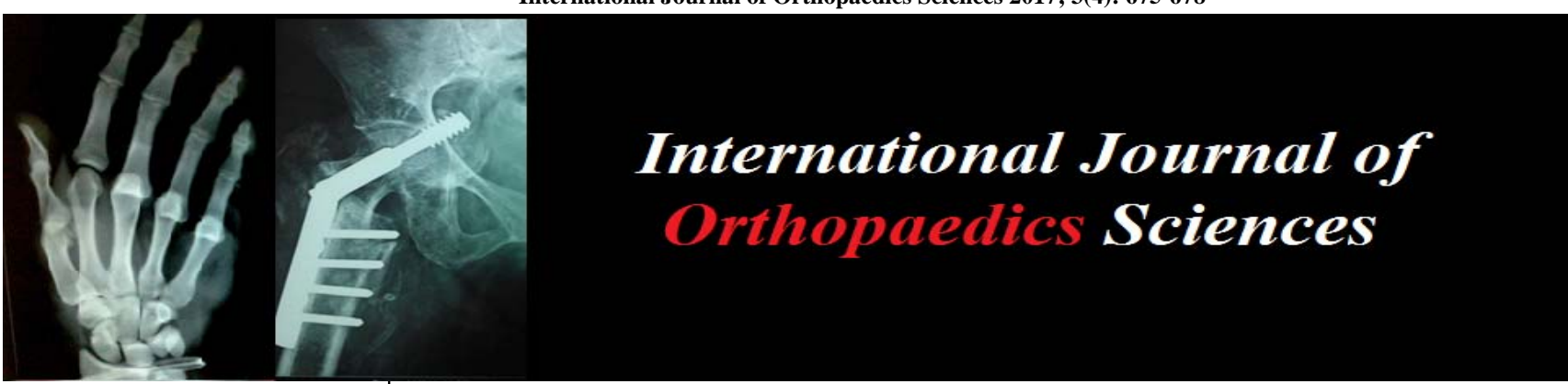

ISSN: $2395-1958$

IJOS 2017; 3(4): 675-678

(C) 2017 IJOS

www.orthopaper.com

Received: 06-08-2017

Accepted: 07-09-2017

Vivek Saxena

DNB, Consultant Orthopaedic

Surgeon; Avadh Hospital,

Jhalod, Gujarat, India

Manoj K. Rajak

MS, Sr. Consultant, Department of Orthopaedics, Tata Main

Hospital. Jamshedpur,

Jharkhand, India

Rajesh Thakur

DNB, Associate Specialist,

Department of Orthopaedics,

Tata Main Hospital.

Jamshedpur, Jharkhand, India

Sachin Kumar

DNB, Associate Specialist,

Department of Orthopaedics,

Tata Main Hospital.

Jamshedpur, Jharkhand, India

Correspondence

Vivek Saxena

DNB; Consultant Orthopaedic

Surgeon; Avadh Hospital,

Jhalod, Gujarat, India

\section{Electrophysiological and functional outcome after carpal tunnel release in patients with carpal tunnel syndrome: A prospective evaluation}

\author{
Vivek Saxena, Manoj K Rajak, Rajesh Thakur and Sachin Kumar \\ DOI: https://doi.org/10.22271/ortho.2017.v3.i4j.93
}

\section{Abstract}

Background: The Carpal tunnel syndrome (CTS) is median nerve entrapment neuropathy at wrist and is one of the commonly diagnosed disabling condition of the upper extremity. The only scientifically established disease modifying treatment is carpal tunnel release (CTR). Electrophysiological study helps in detecting and aiding in the diagnosis of CTS and helps in determining level of improvement after release.

Material and Methods: Two year Prospective Study conducted in Tata Main Hospital, Jamshedpur on 33 cases of carpal tunnel syndrome. All patients underwent open carpel tunnel release. Electrophysiological and functional outcome was assessed using Nerve Conduction Study (NCS) and Boston Carpal Tunnel Questionnaire (BCTQ) both preoperatively and at 1 month, 3 months and 6 months postoperatively.

Results: The CTS was most common in age group 40-50 years. Females were more affected with the ratio of 7:1. Mean BCTQ score preoperatively was 2.9. Post-operative at 1 month it showed marked improvement in mean score to 1.83 . At 3 and 6 month follow up it reduced to 1.58 and 1.51 respectively. NCS were assessed using mean values of Nerve sensory latency (NSL), Nerve Motor Latency (NML), Nerve Sensory Velocity (NSV), and Nerve Motor Velocity (NMV). Preoperative evaluation shows higher latency (NSL 5.23 \pm 0.88 , NML 5.23 \pm 0.63 ) and lower velocity (NSV 30.12 \pm 3.09 , NMV $30.28 \pm 4.30$ ). Both of which improved following CTR with mean NSL 4.46 \pm 0.59 , NML 4.72 \pm 0.47 , NSV $39.55 \pm 1.93$, and NMV $36.55 \pm 5.32$ at 1 month. Further follow up at 3 and 6 month also showed significant improvement.

Conclusions: Boston Carpal Tunnel Questionnaire is good tool to evaluate functional outcome after carpal tunnel release. Nerve Conduction Studies serve as a valuable tool to assess follow up as it can provide reassurance to the patient that their operation is successful and that there is further potential for clinical improvement.

Keywords: Carpal tunnel syndrome, Carpal Tunnel Release

\section{Introduction}

Carpal Tunnel Syndrome (CTS) is the most common and prevalent type of peripheral entrapment neuropathy that accounts for about $90 \%$ of all entrapment neuropathies ${ }^{[1,2]}$ In 2007, the American Academy of Orthopaedic Surgeons (AAOS) created a clinical practice guideline that defined CTS as a symptomatic compression neuropathy of the median nerve at the level of the wrist, characterized physiologically by evidence of increased pressure within the carpal tunnel and decreased function of the median nerve at that level ${ }^{[3]}$.

The peak age of development for CTS is 45-60 years with an estimated $10 \%$ of people with CTS younger than 31 years ${ }^{[4,5]}$.

Gold standard for diagnosis is the combination of the clinical findings and the electrophysiological study ${ }^{[6,7]}$ Diagnostic criteria for CTS in nerve conduction studies (NCS) include the median nerve showing extended amounts of sensory and motor latencies as well as diminished sensory and motor conduction velocities ${ }^{[1]}$. The sensitivity and specificity of a nerve conduction monitoring system help in detecting and aiding in the diagnosis of CTS and is useful in the long-term management of patients with CTS by aiding in determining the level of improvement in median nerve function after carpal tunnel release (CTR) ${ }^{[8]}$. 
Treatment decisions on carpal tunnel syndrome are based on the severity of the symptoms. Non-surgical treatments are recommended for patients with mild symptoms of CTS. Patients with moderate to severe symptoms like persistent numbness and pain after more than 3 months of conservative treatment, motor dysfunction with diminished grip or pinch are recommended for surgical management. Patients achieved a clinical relief along with a significant improvement of electrophysiological parameters such as median sensory latency, sensory nerve conduction velocity and median distal motor latency following Carpal tunnel release ${ }^{[9,10]}$. Levine et al introduced the first disease-specific questionnaire, the Boston Carpal Tunnel Questionnaire (BCTQ), to assess the outcome after carpal tunnel release [11]. There occurs symptomatic relief and functional improvement in BCTQ following carpal tunnel release ${ }^{[12,13]}$.

\section{Aims and Objectives}

To evaluate the electrophysiological outcome by using nerve conduction studies and functional outcome by using selfadministered Boston Carpal Tunnel Questionnaire in patients of carpal tunnel syndrome after carpal tunnel release in a follow up period of 6 months.

\section{Material and method}

We conducted two year prospective study of 33 patients with CTS at Tata Main Hospital, Jamshedpur (from $1^{\text {st }}$ November 2012 to $31^{\text {st }}$ October 2014). Approval from ethical committee of Tata main hospital, Jamshedpur was taken before embarking on the study. Initially clinical diagnosis of CTS was made preliminarily based on symptoms and findings on physical examination. Then we evaluated the patient using self-administered BCTQ and NCS. The parameters used in NCS were sensory and motor latency of median nerve and sensory and motor conduction velocity of median nerve respectively. After preoperative evaluation, all the patients included in the study had open carpal tunnel release under general anaesthesia with upper-arm tourniquet. After surgery, all the patients were evaluated at 1 month, 3 month and 6 month using BCTQ and NCS by the same examiner.

- Inclusion criteria: Diagnosed cases of carpal tunnel syndrome.
- Exclusion criteria:

1. Inability to complete a self-administered BQTS and to undergo NCS.

2. Previous carpal tunnel release.

3. Fracture dislocation around wrist.

4. Associated connective tissue disorders, cervical disc diseases and peripheral vascular disease.

- Parameters to be studied:

1. Demographic parameters (Age, Sex).

2. Electrophysiological study - Nerve conduction studies (NCS).

3. Boston Carpal Tunnel Questionnaire (BCTQ).

- Statistical analysis: All the data has been selected in quantitative way, tabulated, and then analyzed with appropriate statistical tools. Data was presented as mean with standard deviation. Mean and standard deviation was calculated and following statistical significance tests applied.

1. Statistical analysis for determining significance level in BCTQ and NCS preoperatively and postoperatively at 1 month, 3 month and 6 month follow up were performed using "paired t-test".

2. For Test of Significance of sex distribution and laterality of affected hand, "Chi - square test $\left\{\chi^{2}-\right.$ Test $\}$ " was used.

\section{Results}

The age range was between $20-80$ years with mean age of 51 years. The incidences of CTS were most common in age group 40-50 years (42.4\%). The incidence of CTS among female patients is $87.8 \%$ (29 patients) in comparison to male which is $12.1 \%$ (4 patients), leading to the ratio of $7.25: 1$. Right hand was involved in 19 patients $(57.6 \%)$ and left in 14 (42.4\%). All patients were right hand dominant.

Table 1 shows mean BCTQ score in preoperative and 1, 3, 6 month postoperative period. Table 2, 3, 4, and 5 shows mean change in NSL (Nerve sensory latency), NSV (Nerve sensory velocity), NML (Nerve motor latency), NMV (Nerve motor velocity) during the study period.

Table 1: Comparison of BCTQ score calculated in patients during the study period

\begin{tabular}{|c|c|c|c|c|}
\hline Parameter & $\begin{array}{c}\text { BCTQ (pre- } \\
\text { operative) }\end{array}$ & $\begin{array}{c}\text { BCTQ (1 month post- } \\
\text { operative) }\end{array}$ & $\begin{array}{c}\text { BCTQ (3 month post- } \\
\text { operative) }\end{array}$ & $\begin{array}{c}\text { BCTQ (6 month post- } \\
\text { operative) }\end{array}$ \\
\hline Mean & 2.94 & 1.83 & 1.58 & 1.51 \\
\hline Standard deviation & 0.58 & 0.69 & 0.72 & 0.69 \\
\hline
\end{tabular}

Table 2: Comparison of NSL values observed during the study period

\begin{tabular}{|c|c|c|c|c|}
\hline Parameter & $\begin{array}{c}\text { NSL (pre- } \\
\text { operative) }\end{array}$ & $\begin{array}{c}\text { NSL (1 month post- } \\
\text { operative) }\end{array}$ & $\begin{array}{c}\text { NSL (3 month post- } \\
\text { operative) }\end{array}$ & $\begin{array}{c}\text { NSL (6 month post- } \\
\text { operative) }\end{array}$ \\
\hline Mean & 5.23 & 4.46 & 3.83 & 3.19 \\
\hline Standard deviation & 0.88 & 0.59 & 0.54 & 0.45 \\
\hline
\end{tabular}

Table 3: Comparison of NSV values observed during the study period

\begin{tabular}{|c|c|c|c|c|}
\hline Parameter & $\begin{array}{c}\text { NSV (pre- } \\
\text { operative) }\end{array}$ & $\begin{array}{c}\text { NSV (1 month post- } \\
\text { operative) }\end{array}$ & $\begin{array}{c}\text { NSV (3 month post- } \\
\text { operative) }\end{array}$ & $\begin{array}{c}\text { NSV (6 month post- } \\
\text { operative) }\end{array}$ \\
\hline Mean & 30.12 & 39.55 & 42.36 & 45.46 \\
\hline Standard deviation & 3.09 & 1.93 & 2.32 & 2.76 \\
\hline
\end{tabular}

Table 4: Comparison of NML values observed during the study period

\begin{tabular}{|c|c|c|c|c|}
\hline Parameter & $\begin{array}{c}\text { NML (pre- } \\
\text { operative) }\end{array}$ & $\begin{array}{c}\text { NML (1 month post- } \\
\text { operative) }\end{array}$ & $\begin{array}{c}\text { NML (3 month post- } \\
\text { operative) }\end{array}$ & $\begin{array}{c}\text { NML (6 month post- } \\
\text { operative) }\end{array}$ \\
\hline Mean & 5.23 & 4.72 & 4.50 & 4.25 \\
\hline Standard deviation & 0.63 & 0.47 & 0.37 & 0.35 \\
\hline
\end{tabular}


Table 5: Comparison of NMV values observed during the study period

\begin{tabular}{|c|c|c|c|c|}
\hline Parameter & $\begin{array}{c}\text { NMV (pre- } \\
\text { operative) }\end{array}$ & $\begin{array}{c}\text { NMV (1 month post- } \\
\text { operative) }\end{array}$ & $\begin{array}{c}\text { NMV (3 month post- } \\
\text { operative) }\end{array}$ & $\begin{array}{c}\text { NMV (6 month post- } \\
\text { operative) }\end{array}$ \\
\hline Mean & 30.28 & 36.55 & 41.83 & 44.71 \\
\hline Standard deviation & 4.30 & 5.32 & 3.88 & 3.55 \\
\hline
\end{tabular}

\section{Discussion}

In this study, patients after surgical release showed significant improvement in NSV and NML. These findings were consistent with study conducted by Federica Ginanneschi et al in $2008^{[14]}$. who found that shortly after CTR; improved NSV and NML is accompanied by changes in compound muscle action potential (CMAP) with further improvement at 6 month post operatively. Similar results were shown in study conducted by Mondelli $\mathrm{M}$ et al $2000^{[15]}$.

NMV and NSV were significantly improved as early as 1 month post operatively and the NSL and NML improve much at 3 month, 6 month respectively. Shurr et al 1986 also found the similar changes \& the reason is that the CTS compression is in the distal part of the median nerve at the carpal tunnel rather than the proximal part in the forearm ${ }^{[16]}$. Mondelli $\mathrm{M}$ et al in 2000 concluded that outcome of surgical release of the carpel tunnel seem to be predictable only on the basis of neurophysiological data not the subjective data.

Boston Questionnaire shows early improvement of median nerve function after releasing of carpal tunnel even within 1 month; following which there is gradual improvement. BCTQ and NCS should be used together to monitor the CTS patient as they are easy, simple to use and complimentary.

\begin{tabular}{|c|c|c|c|c|c|c|c|c|c|c|c|c|c|c|c|c|c|c|c|c|c|c|c|c|}
\hline S.no & Name & Age & Sex & Laterality & BCTQ & NSL & NSV & NML & NMV & BCTQ & NSL & NSV & NML & NMV & BCTQ & NSL & NSV & NML & NMV & BCTQ & NSL & NSV & NML & NMV \\
\hline 1 & $\mathrm{BD}$ & 40 & $\mathrm{~F}$ & Left & 2.42 & 4.14 & 36.2 & \begin{tabular}{|l|}
4.7 \\
\end{tabular} & 32.6 & 1 & 3.92 & 38.2 & 4.52 & 46.6 & 1 & 2.68 & 43.2 & \begin{tabular}{|l|}
4.44 \\
\end{tabular} & 46.6 & 1 & 2.6 & 44.2 & \begin{tabular}{|l|}
3.8 \\
\end{tabular} & 46.6 \\
\hline 2 & DD & 61 & $\mathrm{~F}$ & Left(b/l) & 2.63 & 4.42 & 29.2 & 4.9 & 38.1 & 1 & 4.14 & 39.4 & 4.79 & 44.3 & 1 & 3.92 & 45.6 & 4.62 & 45.2 & 1 & 3.56 & 46.2 & 3.94 & 45.9 \\
\hline 3 & MSD & 48 & $\mathrm{~F}$ & Right & 2.52 & 5.72 & 34.6 & 4.53 & 29.6 & 1 & 4.44 & 39.6 & 4.01 & 34.2 & 1 & 2.96 & 43.9 & 3.97 & 39.8 & 1 & 2.82 & 45.3 & 3.45 & 47.3 \\
\hline 4 & AS & 45 & $\mathrm{~F}$ & Right & 2.84 & 4.1 & 32.6 & 4.61 & 22.8 & 1.68 & 3.68 & 40.2 & 4.27 & 36.2 & 1.47 & 2.94 & 44.3 & 4.04 & 36.9 & 1.42 & 2.43 & 46.3 & 3.82 & 42.5 \\
\hline 5 & FD & 52 & $\mathrm{~F}$ & Right & 3.15 & 4.63 & 27.6 & 4.87 & 31.5 & 1.89 & 4.26 & 38.2 & 4.53 & 39.1 & 1.78 & 3.46 & 39.9 & 4.16 & 40 & 1.68 & 3.14 & 42.3 & 3.98 & 47.8 \\
\hline 6 & SD & 42 & $\mathrm{~F}$ & Right & 2.57 & 5.8 & 32.4 & 4.9 & 34.2 & 2.8 & 4.62 & 39.3 & 4.67 & 41.1 & 1.15 & 3.54 & 42.5 & 4.29 & 41.6 & 1.15 & 3.23 & 45.3 & 4.14 & 46.9 \\
\hline 7 & JD & 52 & $\mathrm{~F}$ & Right & 2.73 & 4.7 & 29.6 & 5.1 & 31.6 & 2.6 & 3.94 & 40.8 & 4.44 & 39.6 & 1.36 & 3.62 & 41.6 & 4.27 & 39.6 & 1.26 & 3.16 & 47.2 & 4.04 & 44.5 \\
\hline 8 & NM & 70 & $\mathrm{~F}$ & Left & 2.94 & 4.61 & 28.6 & 5.32 & 37.4 & 2.8 & 3.8 & 38.5 & 4.16 & 41.3 & 1.21 & 3.26 & 40.6 & 3.98 & 41.4 & 1.15 & 3.23 & 44.3 & 3.92 & 42.6 \\
\hline 9 & JLD & 81 & $\mathrm{M}$ & Right & 3.05 & 5.58 & 32.1 & 4.98 & 29.5 & 1.89 & 4.16 & 39.2 & 4.24 & 36.8 & 1.68 & 3.92 & 40.9 & 4.04 & 40.8 & 1.68 & 2.84 & 43.9 & 3.98 & 44.5 \\
\hline 10 & $\mathrm{KS}$ & 41 & $\mathrm{~F}$ & Right & 3.05 & 5.92 & 32.7 & 5.2 & 30.2 & 2.1 & \begin{tabular}{|l|}
4.84 \\
\end{tabular} & 38.3 & 4.82 & 36.5 & 2 & 4.26 & 39.5 & 4.71 & 38.4 & 2 & 3.24 & 44.9 & 4.56 & 40.9 \\
\hline 11 & DD & 61 & $\mathrm{~F}$ & Left & 2.57 & 4.81 & 29.4 & 4.9 & 29.3 & 1.57 & 3.64 & 41.3 & 4.34 & 34.8 & 1.26 & 3.14 & 45.1 & 4.18 & 36.4 & 1.21 & 2.98 & 47.9 & 4.02 & 40.8 \\
\hline 12 & MD & 42 & $\mathrm{~F}$ & Right & 2.84 & 4.96 & 30.8 & 5.2 & 28.6 & 1.78 & 3.82 & 41.3 & 4.98 & 38.1 & 1.57 & 3.6 & 44.8 & 4.62 & 38.4 & 1.47 & 2.96 & 46.5 & 4.34 & 39.4 \\
\hline 13 & VJ & 44 & $\mathrm{~F}$ & ight & 2.94 & 5.23 & 27.5 & 4.3 & 27.5 & 1.47 & \begin{tabular}{|l|}
4.67 \\
\end{tabular} & 42.5 & 4.22 & 32.5 & 1.36 & 4.26 & 46.8 & 4.19 & 34.2 & 1.21 & 3.14 & 47.2 & 4.09 & 39.5 \\
\hline 14 & PP & 35 & $\mathrm{~F}$ & Right & 3.26 & 4.97 & 24.6 & 6.2 & 31.9 & 1.36 & \begin{tabular}{|l|}
4.17 \\
\end{tabular} & 41.6 & 4.97 & 39.8 & 1.26 & 5.14 & 43.7 & 4.74 & 40.5 & 1.1 & 4.49 & 44.9 & 4.54 & 41.1 \\
\hline 15 & SD & 73 & $\mathrm{~F}$ & ight & 4.21 & \begin{tabular}{|l|}
7.2 \\
\end{tabular} & 26.3 & 6.5 & 30.2 & 3.57 & 6.42 & 36.9 & 5.42 & 36.2 & 3.47 & 4.29 & 39.4 & 4.9 & 38.1 & 3.31 & 4 & 41.3 & 4.72 & 41.8 \\
\hline 16 & JVSL & 43 & $\mathrm{~F}$ & Right & 2.57 & 4.68 & 31.2 & 5.1 & 28.5 & 1.42 & 4.62 & 43.2 & 4.75 & 32.5 & 1.36 & 3.53 & 44.9 & 4.56 & 36.1 & 1.31 & 3.26 & 47.2 & 4.24 & 40.5 \\
\hline 17 & SS & 60 & $\mathrm{~F}$ & & 3.26 & 6.48 & 32.8 & 4.69 & 29.7 & 2.57 & \begin{tabular}{|l|}
4.34 \\
\end{tabular} & 39.5 & 4.19 & 31.5 & 2.42 & 3.97 & 43.1 & 4.04 & 39.4 & 2.36 & 3.47 & 45.3 & 3.97 & 42.4 \\
\hline 18 & PS & 33 & $\mathrm{~F}$ & Left & 2.31 & 4.65 & 28.3 & 4.87 & 26.5 & 1.47 & \begin{tabular}{|l|}
4.26 \\
\end{tabular} & 43.1 & 4.24 & 29.4 & 1.31 & 3.84 & 45.8 & 4.18 & 40.9 & 1.31 & 3.22 & 47.3 & 3.99 & 43.5 \\
\hline 19 & RBD & 41 & $\mathrm{~F}$ & & 4.52 & 4.2 & 25.4 & 4.99 & 20.8 & 3.68 & \begin{tabular}{|l|}
3.89 \\
\end{tabular} & 35.2 & 4.54 & 25.3 & 3.68 & 3.61 & 37.4 & 4.62 & 44.5 & 3.52 & 3.05 & 52.5 & 4.42 & 49 \\
\hline 20 & MD & 48 & $\mathrm{~F}$ & Right & 2.73 & 4.25 & 30.6 & 5.3 & 26.5 & 1.42 & 4.16 & 40.2 & 4.82 & 32.7 & 1.31 & 3.97 & 41.3 & 4.64 & 46.9 & 1.21 & 3.42 & 43.2 & 4.39 & 46.7 \\
\hline 21 & $\mathrm{AK}$ & 59 & $\mathrm{~F}$ & eft & 2.47 & 6.58 & 31.8 & 5.2 & 32.9 & 1.47 & \begin{tabular}{|l|}
4.34 \\
\end{tabular} & 40.3 & 5.06 & $36 . .4$ & 1.42 & 3.14 & 43.8 & 4.94 & 48.7 & 1.21 & 2.62 & 46.1 & 4.72 & 48.9 \\
\hline 22 & ND & 49 & $M$ & Left & 3.78 & 6.54 & 24.2 & 6.3 & 21.8 & 2.36 & 5.21 & 39.5 & 5.49 & 28.9 & 2.26 & 4.72 & 42.6 & 4.86 & 48.5 & 2.26 & 3.76 & 43.8 & 4.64 & 48.6 \\
\hline 23 & RT & 52 & $\mathrm{~F}$ & Left & 4.36 & 7.46 & 23.8 & 7.1 & 27.6 & 3.26 & 5.34 & 34.9 & 5.92 & 29.6 & 3.15 & 4.16 & 39.2 & 4.98 & 41.9 & 2.84 & 3.6 & 41.2 & 4.79 & 41.9 \\
\hline 24 & $\mathrm{SS}$ & 20 & $\mathrm{~F}$ & Right & 2.63 & 4.62 & 30.6 & 4.3 & 31.8 & 1.47 & 4.16 & 38.9 & 4.12 & 33.6 & 1.36 & 3.98 & 39.4 & 4.01 & 39.4 & 1.26 & 2.74 & 43.1 & 3.92 & 41.5 \\
\hline 25 & LD & 47 & $\mathrm{~F}$ & Right & 3.78 & 4.15 & 26.3 & 5.6 & 23.2 & 2.73 & 5.12 & 37.2 & 4.94 & 28.4 & 2.52 & 4.72 & 40.3 & 4.74 & 47.9 & 2.47 & 3.12 & 41.3 & 4.19 & 47.9 \\
\hline 26 & RK & 62 & $\mathrm{~F}$ & Left & 2.42 & 5.88 & 31.2 & 5.23 & 31.3 & 1.21 & 4.98 & 40.3 & 4.87 & 36.2 & 1 & 3.78 & 42.3 & 4.42 & 39.5 & 1 & 3.16 & 44.8 & 4.27 & 42.7 \\
\hline 27 & PD & 52 & $\mathrm{~F}$ & Right & 2.57 & 4.99 & 32.8 & 5.47 & 30.4 & 1.31 & 5.22 & 39.4 & 5.14 & 35.8 & 1.1 & 3.98 & 41.9 & 5.01 & 40.7 & 1.1 & 3.26 & 43.8 & 4.83 & 40.9 \\
\hline 28 & PM & 52 & $\mathrm{~F}$ & Right & 2.73 & 5.25 & 31.6 & 4.69 & 31.2 & 1.47 & \begin{tabular}{|l|}
4.16 \\
\end{tabular} & 41.9 & 4.42 & 39.1 & 1.15 & 3.78 & 44.2 & 4.39 & 44.9 & 1.1 & 3.45 & 45.9 & 4.06 & 49.5 \\
\hline 29 & $\mathrm{MC}$ & 45 & $\mathrm{~F}$ & Right & 2.31 & 4.89 & 29.6 & 5.36 & 36.6 & 1.26 & \begin{tabular}{|l|}
4.99 \\
\end{tabular} & 39.6 & 5.24 & 39.4 & 1.1 & 4.32 & 45 & 5.12 & 42.7 & 1.05 & 3.96 & 45.8 & 4.88 & 44.8 \\
\hline 30 & NJB & 57 & $\mathrm{~F}$ & Left & 2.52 & 5.65 & 30.8 & 5.47 & 33.5 & 1.15 & 4.34 & 40.9 & 4.87 & 37.2 & 1.05 & 4.07 & 39.5 & 4.65 & 47.4 & 1 & 2.82 & $41 . .8$ & 4.19 & 50.1 \\
\hline 31 & KNS & 49 & $M$ & Left & 2.57 & 4.78 & 32.6 & 5.98 & 38.4 & 1.15 & 4.62 & 37.8 & 5.62 & 42.6 & 1.05 & 4.09 & 40.9 & 5.32 & 42.4 & 1 & 2.55 & 52.7 & 4.73 & 53.5 \\
\hline 32 & SD & 64 & $\mathrm{~F}$ & Left & 2.89 & 5.55 & 34.4 & 5.69 & 30.8 & 1.26 & 4.98 & 39.7 & 4.26 & 46.3 & 1.1 & 4.23 & 41.8 & 4.12 & 46.7 & 1 & 2.78 & 50.1 & 4.09 & 46.8 \\
\hline 33 & MSD & 52 & $\mathrm{M}$ & Right & 2.73 & 5.3 & 31.6 & 5.01 & 32.6 & 1.36 & 3.82 & 38.4 & 4.94 & 43.9 & 1.1 & 3.56 & 42.6 & 4.64 & 43.9 & 1.05 & 3.12 & 42.9 & 4.47 & 44 \\
\hline
\end{tabular}

Abbreviations

BCTQ - Boston Carpal Tunnel Questionnaire

NSL - Nerve Sensory Latency

NSV - Nerve Sensory Velociy

NML - Nerve Motor Latency

NMV - Nerve Motor Velocity

\section{Conclusion}

In our study we found that Boston Questionnaire due to its ease of administration, reproducibility, internal consistency, validity and responsiveness to clinical change can be considered as a valuable tool to evaluate functional outcome. With respect to nerve conduction studies, CTS patients had higher sensory and motor latency values in preoperative period, which decreased at 1 month post-operative period; and further gradually improved in 3 month and 6 month follow up. CTS patients also showed lower sensory and motor conduction velocity which gradually improved in follow up periods of 6 month.

\section{References}

1. Ibrahim I, Khan WS, Goddard N, Smitham P. Carpal tunnel syndrome: a review of the recent literature. Open Orthop J. 2012; 6:69-76.

2. Mashoof AA, Levy HJ, Soifer TB, Miller-Soifer F, Bryk E, Vigorita V. Neural anatomy of the transverse carpal ligament. Clin Orthop Relat Res. 2001; 386:218-21.

3. American Academy of Orthopaedic Surgeons Work Group Panel. Clinical guidelines on 
diagnosisofcarpaltunnelsyndrome. Availableatwww. aaosorg/research/guidelines/CTS_guideline.pdf.

4. De Krom MC, Kester AD, Knipschild PG, Spaans F. Risk factors for carpal tunnel syndrome. Epidemiology. 1990; 132(6):1102-10.

5. Nordstrom DL, DeStefano F, Vierkant RA, Layde PM. Incidence of diagnosed carpal tunnel syndrome in a general population. Epidemiology. 1998; 9(3):342-5.

6. Kohara N. Clinical and electrophysiological findings in carpal tunnelsyndrome. Brain Nerve. 2007; 59:1229-38.

7. Robinson, L. Electrodiagnosis of Carpal Tunnel Syndrome. Phys Med RehabilClin N Am. 2007; 18(4):733-46.

8. Rotman MB, Enkvetchakul BV, Megerian JT, GozaniSNTime course and predictors of median nerve conduction after carpal tunnel release. J Hand Surg Am. 2004; 29(3):367.

9. El-Hajj T, Tohme R, Sawaya R. Changes in electrophysiological parameters after surgery for the carpal tunnel syndrome. J Clin Neurophysiol. 2010; 27(3):224-6.

10. Ji Won Yang, Young Hee Sung, KeeHyung Park, Yeong Bae Lee, Dong Jin Shin, HyeonMi Park. Clinical and Electrophysiological Changes after Open Carpal Tunnel Release: Preliminary Study of 25 Hands. Korean J Clin Neurophysiol. 2014; 16(1):21-26.

11. Levine DW, Simmons BP, Koris MJ, Daltroy LH, Hohl $\mathrm{GG}$, Fossel AH et al. A self-administered questionnaire for the assessment of severity of symptoms and functional status in carpal tunnel syndrome. J Bone Joint Surg Am. 1993; 75(11):1585-1592.

12. Glowacki KA, Breen CJ, Sachar K, Weiss AP. Eletrodiagnostic testing and carpal tunnel release outcome. J Hand Surg Am. 1996; 21:117-22.

13. Stephen A Badger, Mark E, O'Donnell, Jagannath M, Sherigar, Peter Connolly et al. Open Carpal Tunnel Release - still a safe and effective operation. Ulster Med J. Jan. 2008; 77(1):22-24.

14. Federica Ginanneschi, Paolo MilaniFabio Reale Alessandro Rossi Short-term electrophysiological conduction change in median nerve fibres after carpal tunnel release. Clini Neurol Neurosurg. 2008; 110(10):1025-30.

15. Mondelli M, Reale F, Sicurelli F, Padua L. Relationship between the self-administered Boston questionnaire and electrophysiological findings in follow-up of surgicallytreated carpal tunnel syndrome. J Hand Surg Br. 2000; 25(2):128-34.

16. Shurr DG, Blair WF, Bassett G. Electromyographic changes after carpel tunnel release. J Hand Sug. 1986; $11: 876-80$. 